\title{
Anomalias dos nervos lombossacrais em natimortos a termo. Estudo anatômico
}

\author{
Max Franco de Carvalho', Roberta Teixeira Rocha², João Tiago Silva Monteiro ${ }^{3}$, \\ Carlos Umberto Pereira ${ }^{4}$, Alex Franco de Carvalho ${ }^{4}$, Francisco do Prado Reis ${ }^{5}$
}

Laboratório de Anatomia do Departamento de Morfologia do Centro de Ciências

Biológicas e da Saúde da Universidade Federal de Sergipe (UFS).

\section{RESUMO}

Contexto: Existe controvérsia entre autores quanto à incidência de variações anatômicas dos nervos espinhais lombossacros. A incidência dessas anomalias nervosas varia de $0 \%$ a $30 \%$ como verificado em estudos anatômicos em cadáveres adultos de diferentes nacionalidades, de 2,0\% a 4,0\% em estudos de imagem diagnóstica e de 0,34\% a 10\% em séries cirúrgicas. Objetivo: Determinar a incidência de anomalias anatômicas nos nervos espinhais lombossacros em 40 natimortos a termo de nacionalidade brasileira. Métodos: O segmento lombossacro da coluna vertebral de 40 natimortos, provenientes do laboratório de patologia clínica do Hospital Universitário da Universidade Federal de Sergipe (UFS), foi estudado no Laboratório de Neuroanatomia do Departamento de Morfologia da UFS. Foram excluídos aqueles casos que possuíam síndromes ou qualquer defeito orgânico associado. Os espécimes anatômicos foram fixados com solução de formaldeído a $10 \%$, infundida por meio da veia umbilical. As peças foram preparadas com a ressecção em bloco das partes moles dorsais, processos espinhosos e lâminas da décima vértebra torácica até o sacro. Resultado: Neste estudo, foram encontradas seis variações anatômicas dos nervos espinhais lombossacros, em seis diferentes espécimes, de quatro tipos, totalizando $15 \%$ das peças. As variações anatômicas dos nervos espinhais lombossacros foram classificadas em oito tipos, sendo a anastomose intradural a mais presente. O quinto nervo lombar foi o mais frequentemente comprometido Não foi observada diferença significativa quanto ao sexo e lado envolvido. Conclusão: O presente estudo enfatiza a importância do conhecimento dessas anomalias anatômicas durante os procedimentos cirúrgicos na região lombossacral, pois não se trata de evento muito raro.

\section{PALAVRAS-CHAVE}

Nervo lombossacral. Anomalias.

\section{ABSTRACT}

Anomalies of the lumbosacral nerve roots in stillborns. An anatomical study

Context: There is such controversy among authors about the real incidence of lumbosacral nerve roots anomalies. The incidence ranges from $0 \%$ to $30 \%$ in anatomic studies in different populations, from $2 \%$ to $4 \%$ in diagnostic image studies and $0.34 \%$ to $10 \%$ in surgical series. Objective: To determine the incidence of anatomical abnormalities of the lumbossacral spinal nerves in 40 Brazilian stillborns. Methods: The segment of the lumbosacral spine of 40 stillborns, from the clinical pathology laboratory at the University Hospital of Federal University of Sergipe (UFS) was studied in the Laboratory of Neuroanatomy, Department of Morphology of the UFS. We excluded those cases that had any defects or syndromes associated. The anatomical specimens were fixed with formaldehyde solution $10 \%$, infused through the umbilical vein. The specimens were prepared with en bloc resection of the soft dorsal spinous processes and laminae from the tenth thoracic vertebra to the sacrum. Results: In this study, were found four types of anatomical variations of lumbosacral spinal nerves in six different specimens, accounting for $15 \%$ of the specimens. The fifth lumbar nerve was the most frequently involved (66\%). The anatomical variations of lumbosacral spinal nerves were classified into eight types, and the intradural anastomosis was the most frequent. There was no significant difference regarding gender and side involved. Conclusion: Our study emphasizes the importance of knowledge of these anatomical anomalies during surgical procedures in the lumbosacral region because they are not too rare.

\section{KEY WORDS}

Lumbosacral nerve roots. Anomaly.

1 Professor MSc substituto do Departamento de Medicina da Universidade Federal de Sergipe (UFS). Aluno do Programa de Pós-graduação (doutorado) da Faculdade de Medicina de Ribeirão Preto da Universidade de São Paulo (FMRP-USP).

2 Médica Radiologista da UFS.

3 Acadêmico de Medicina e bolsista PIBIC (01220587) UFS.

4 Professor Doutor Adjunto do Departamento de Medicina da UFS.

5 Professor Titular do Departamento de Morfologia da UFS. 


\section{Introdução}

As descrições clássicas da coluna vertebral nos livros de anatomia não fazem referência sobre a existência de variações anatômicas na origem ou trajeto dos nervos espinhais lombossacros. ${ }^{6,8,21}$ A maioria dos estudos aborda essas anomalias por meio de relatos de casos, pequenas séries de achados incidentais transoperatórios e de exames imagéticos. $3,5,9,10,16,17,20$

Existe controvérsia entre autores quanto à incidência de variações anatômicas dos nervos espinhais lombossacros. A incidência dessas anomalias nervosas varia de $0 \%$ a $30 \%$ como verificado em estudos anatômicos em cadáveres adultos de diferentes nacionalidades, ${ }^{2,7,9,13} \mathrm{de}$ $2,0 \%$ a $4,0 \%$ em estudos de imagem ${ }^{9,14,16}$ e de $0,34 \%$ a $10 \%$ em séries cirúrgicas. ${ }^{4,15}$

Os relatos cirúrgicos de que os nervos espinhais anômalos ocupam mais espaço no canal vertebral ou forâmen intervertebral e a dificuldade de mobilização transoperatória desses nervos sugerem que haveria sintomas clínicos compressivos lombares, durante os movimentos normais de flexão e extensão da coluna vertebral, sem haver a necessidade de compressão extrínseca. ${ }^{10,22}$

Por muito tempo, a importância clínica dessas anomalias estava relacionada aos maus resultados pós-discectomia e lombalgias de causas obscuras. Postacchini et al. ${ }^{16}$, com base em estudo de 46 casos com anormalidades anatômicas dos nervos espinhais lombossacros, diagnosticadas por meio de mielografia, concluíram que essas anomalias são achados incidentais sem qualquer importância clínica.

Em razão do grande número de procedimentos cirúrgicos na região lombossacra, o conhecimento dessas anomalias anatômicas é de grande importância, para evitar lesões iatrogênicas e danos neurológicos irreparáveis. O objetivo deste estudo é determinar a incidência de anomalias anatômicas nos nervos espinhais lombossacros em 40 natimortos a termo de nacionalidade brasileira.

\section{Casuística e métodos}

O segmento lombossacro da coluna vertebral de 40 natimortos ( 21 masculinos e 19 femininos), provenientes do laboratório de patologia clínica do Hospital Universitário da Universidade Federal de Sergipe (UFS), foi estudado no Laboratório de Neuroanatomia do Departamento de Morfologia da UFS. Foram excluídos aqueles casos que possuíam síndromes ou qualquer defeito orgânico associado. Os espécimes anatômicos foram fixados com solução de formaldeído a $10 \%$, infundida por meio da veia umbilical. As peças foram preparadas com a ressecção em bloco das partes moles dorsais, processos espinhosos e lâminas da décima vértebra torácica até o sacro. $\mathrm{O}$ estudo extradural consistiu na observação macroscópica da simetria, da origem, do trajeto, da emergência dural dos nervos espinhais até o forâmen de conjugação e relação dos nervos espinhais com os pedículos. Após o estudo extradural, a dura-máter foi aberta longitudinalmente, permitindo a visualização direta da medula espinhal, origem dos filamentos radiculares na medula espinhal, cone medular, cauda equina e junção intradural dos filamentos radiculares que formam as raízes nervosas ventrais e dorsais.

Os fragmentos intradurais e extradurais obtidos durante o estudo macroscópico foram retirados e fixados em formol a $10 \%$ por 48 horas e, após esse período, inclusos em parafina e realizados cortes transversais de 5 micrômetros e estudados histologicamente pela técnica de hematoxilina e eosina.

A incidência de anomalias nervosas na população estudada foi comparada com outras investigações anatômicas pelo teste de qui-quadrado.

\section{Resultados}

O estudo anatômico revelou seis (15\%) anormalidades anatômicas dos nervos lombossacros em seis diferentes espécimes. Dessas, três foram intradurais e três extradurais. Das intradurais, duas anastomoses intradurais estavam entre as raízes do nervo dorsal (Figura 1) e uma entre as raízes do nervo ventral. Nesses três casos, foi observada uma camada espessa da aracnoide aderida aos filamentos radiculares que dificultava a mobilização e individualização dessas raízes. Nas anomalias extradurais, foram visualizadas uma emergência dural comum de duas raízes nervosas (Figura 2), uma divisão extradural e uma raiz nervosa com origem dural cranial que emergia pelo forâmen intervertebral cranial ao esperado (Tabela 1).

A raiz de L5 esteve envolvida em quatro casos (66\% dos casos) e S1 em três (50\%). Três espécimes foram do sexo masculino e três do feminino, os lados direito e o esquerdo foram acometidos em três casos cada.

Durante o estudo das três anomalias intradurais, o conteúdo intradural estava friável, o que impediu um estudo detalhado da organização intradural. O estudo histológico revelou tecido nervoso em todas as raízes nervosas anômalas. 

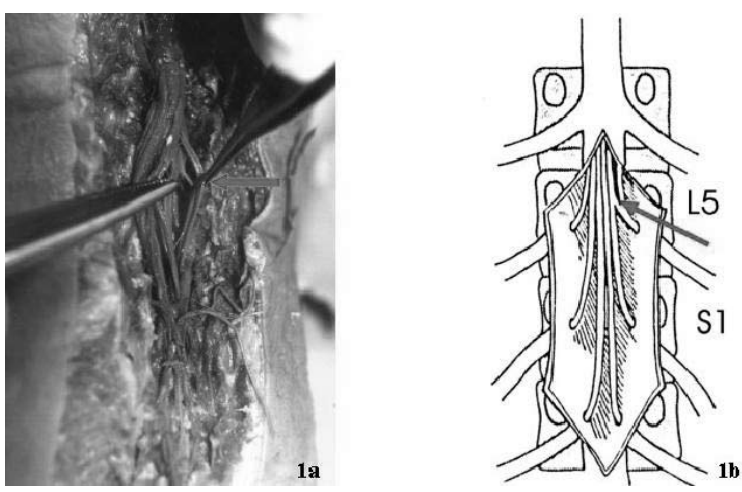

Figura 1 - Anastomose intradural entre L5-S1 em raiz nervosa dorsal direita. 1a. Fotografia do espaço intradural dorsal; a seta demonstra anomalia do tipo I, com anastomose entre a raiz dorsal do quinto nervo espinhal lombar direito e a raiz dorsal do primeiro nervo sacro. 1 b. Ilustração esquemática do espaço intradural dorsal; a seta demonstra anomalia do tipo I, com anastomose entre a raiz dorsal do quinto nervo espinhal lombar direito e a raiz dorsal do primeiro nervo sacro.
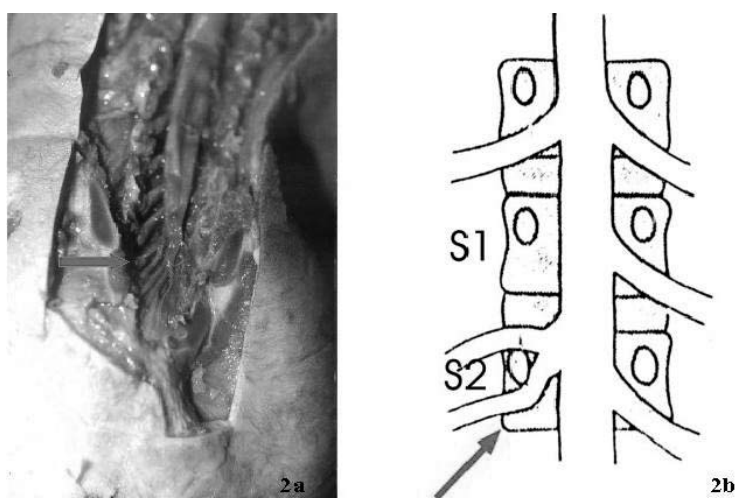

Figura 2-Emergência dural comum S1-S2 em raiz nervosa esquerda. 2a. Fotografia do canal vertebral dorsal; a seta demonstra anomalia do tipo IV entre o primeiro e segundo nervos espinhais sacros esquerdos. $2 b$. Ilustração esquemática do canal vertebral dorsal, onde a seta demonstra anomalia do tipo IV entre o primeiro e segundo nervos espinhais sacros esquerdos.

\begin{tabular}{|c|c|c|c|c|}
\hline \multicolumn{5}{|c|}{$\begin{array}{c}\text { Tabela } 1 \\
\text { Anomalias das raízes encontradas }\end{array}$} \\
\hline Feto $\mathrm{n}^{\circ}$ & Tipo I & Tipo IV & Tipo V & Tipo VII \\
\hline 07 & & $\begin{array}{l}\text { S1 e S2 } \\
\text { esquerda }\end{array}$ & & \\
\hline 17 & $\begin{array}{l}\text { L5 e S1 } \\
\text { esquerda }\end{array}$ & & & \\
\hline 23 & $\begin{array}{l}\text { L5 e S1 } \\
\text { direita }\end{array}$ & & & \\
\hline 24 & & & & $\begin{array}{c}\text { L4 } \\
\text { esquerda }\end{array}$ \\
\hline 32 & & & $\begin{array}{l}\text { L5 } \\
\text { direita }\end{array}$ & \\
\hline 38 & $\begin{array}{c}\text { L4 e L5 } \\
\text { direita }\end{array}$ & & & \\
\hline
\end{tabular}

\section{Discussão}

De acordo com os estudos de Kadish e Simmons ${ }^{9}$ e de Chotigavanich e Sahatapes, ${ }^{2}$ existem oito tipos de anomalias:1,11,12,17,22 tipo I - anastomose intradural; tipo II - anastomose extradural; tipo III - emergência assimétrica (a) cranial, (b) caudal; tipo IV - emergência dural comum; tipo $\mathrm{V}$ - divisão extradural (raízes dorsais e ventrais originam dois nervos distintos); tipo VI - saco dural termina no nível das vértebras lombares com os nervos sacrais, originando-se de um tronco nervoso único; tipo VII - o nervo deixa o canal vertebral por meio do forâmen vertebral caudal ou cefálico; tipo VIII) complexa (Figura 3).
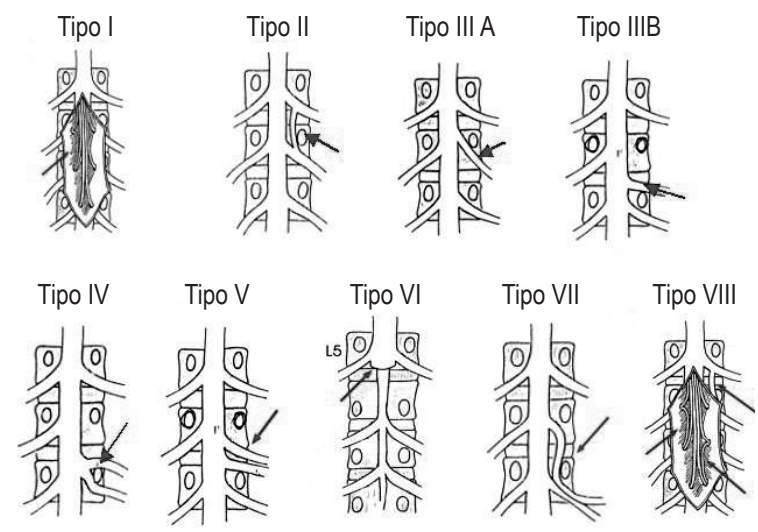

Figura 3 - Ilustração esquemática da classificação, com setas demonstrando as anomalias dos nervos espinhais lombossacros. Tipo I: anastomose intradural. Tipo II: anastomose extradural. Tipo IIIa: emergência assimétrica cranial. Tipo IIIb: emergência assimétrica caudal. Tipo IV: emergência dural comum. Tipo V: divisão extradural (raízes dorsais e ventrais originam dois nervos distintos). Tipo VI: saco dural termina no nível das vértebras lombares com os nervos sacrais, originando-se de um tronco nervoso único. Tipo VII: o nervo deixa o canal vertebral por meio do forâmen vertebral caudal ou cefálico. Tipo VIII: complexa.

A incidência de variações anatômicas dos nervos espinhais lombossacros encontrada neste estudo foi de 15\%. Os achados não apresentaram discrepância significativa $(p=0,87)$ em relação ao estudo anatômico de Kadish e Simmons, ${ }^{9}$ que observaram $14 \%$ em população norte-americana. Entretanto, houve diferença significante $(\mathrm{p}=0,04)$ quando comparado com a incidência de $30 \%$ encontrada no estudo anatômico realizado por Chotigavanich e Sahatapes ${ }^{2}$ em cadáveres adultos de origem tailandesa, e com a de Oliveira e cols., ${ }^{13}$ que não observaram nenhuma anormalidade anatômica nos nervos espinhais após dissecar o conteúdo extradural e intradural de 35 colunas de cadáveres adultos brasileiros. Hasue e cols. ${ }^{7}$ relataram uma incidência de $8,5 \%$ de variações anatômicas extradurais dos nervos espinhais lombossacros, porém não realizaram o estudo intradural das raízes ventrais e dorsais dos nervos espinhais durante a avaliação anatômica. 
Os achados do presente estudo estão de acordo com a literatura quanto à inexistência de predominância quanto ao sexo e ao lado acometido. ${ }^{2,7,9}$ A presença de deformidades congênitas da coluna vertebral associada com anomalias dos nervos espinhais tem sido relatada na literatura. ${ }^{1,2,9,11,12,18,19,22}$ Entretanto, essa associação não foi por nós estudada.

A anomalia tipo I foi responsável por $50 \%$ dos casos em questão, as anomalias tipos IV, V e VII foram observadas em apenas um caso cada. Nos estudos cirúrgicos de White $3^{\text {rd }}$ e cols., ${ }^{22}$ Neidre e Macnab ${ }^{12}$ e Stambough e cols. ${ }^{20}$, a emergência dural comum dos nervos espinhais (tipo IV) foi a mais frequentemente encontrada.

Nas variações anatômicas descritas por Kadish e Simmons, ${ }^{9}$ as anomalias mais presentes foram as dos tipos I e IV. Já no estudo de Chotigavanich e Sahatapes, ${ }^{2}$ a anomalia anatômica do tipo VIII (complexa) foi a mais frequente. Apesar de essa anomalia não ter sido observada no presente estudo, vale ressaltar que em todos os casos classificados como tipo VIII havia anastomose intradural entre os nervos espinhais envolvidos.

$\mathrm{O}$ quinto nervo lombar foi o mais envolvido. Por causa do grande número de procedimentos cirúrgicos na coluna lombar baixa, o conhecimento dessas anormalidades pode evitar lesões iatrogênicas durante procedimentos e descompressão insuficiente do canal vertebral. Essas anomalias devem ser lembradas sempre que houver uma discordância entre o exame físico e os achados de diagnóstico por imagem. Durante o ato cirúrgico, a dificuldade de mobilização dos nervos espinhais ou a presença local de compressão radicular ou medular mínima para um quadro clínico exuberante, também, podem constituir fortes indícios da presença de anormalidades anatômicas.

\section{Conclusão}

Variações anatômicas dos nervos espinhais lombossacros foram achados frequentes no presente estudo. É importante relacionar a presença de anomalia dos nervos espinhais sempre que houver discrepância entre o exame físico e os achados de imagem e quando houver dificuldade de mobilização das raízes nervosas em procedimentos cirúrgicos no canal vertebral.

\section{Referências}

1. Cannon BW, Hunter SE, Picasa JA. Nerve roots anomalies in lumbar disc surgery. J Neurosurg. 1962;9:208-14.
2. Chotigavanichc C, Sahatapes S. Anomalies of the lumbosacral nerve roots: an anatomic investigation. Clin Orthop Rel Res. 1992;278:46-50.

3. Epstein JA, Canas R, Ferrar J, Hyman RA, Khan A. Conjoined lumbosacral nerve roots. J Neurosurg. 1981;55:585-9.

4. Ethelberg S, Riishede J. Malformation of lumbar spinal roots and sheaths in the causation of low backache and sciatica. J Bone Joint Surg Br. 1952;34-B:442-6.

5. Goffin J. Plets C. Association of conjoined and anastomoses nerve roots in the lumbar region. Clin Neurol Neurosurg. 1987;89:117-20.

6. Goss GH. Anatomia de Gray. $29^{\text {th }}$ ed. Rio de Janeiro: Guanabara Koogan; 1988. p. 806-19.

7. Hasue M, Kikuchi S, Sakuyama Y, Yto T. Anatomic study of the interralation between lumbosacral nerve roots and their surroundings tissues. Spine. 1983;8:50-8.

8. Hollinshead W. Anatomy for surgeons. $3^{\text {rd }}$ ed. New York: Harper \& Row; 1982. v. 3., p.19-23.

9. Kadish LJ, Simmons EH. Anomalies of the lumbosacral nerve roots. An anatomical investigation and myelographic study. J Bone Joint Surg Br. 1984;66:411-6.

10. Keon-Cohen B. Abnormal arrangement of the lower lumbar and first sacral nerves within the spinal canal. J Bone Joint Surg Br. 1968;50:261-5.

11. Maiuri F, Gambardella A. Anomalies of the lumbosacral nerve roots. Neurol Res. 1989;11:130-5.

12. Neidre A, Macnab I. Anomalies of the lumbosacral nerve roots: review of 16 cases and classification. Spine. 1983;8:294-9.

13. Oliveira RP, Rodrigues NR, Galvão PEC, Barros Filho TEP. Anomalias das raízes nervosas lombares: estudo anatômico. Acta Ortop Bras. 1996;4:1-3.

14. Peyester RG, Treplic JG, Hashin ME. Computed tomography of lumbosacral conjoined nerve roots anomalies. Potencial cause of false positive reading for herniated nucleus pulposus. Spine. 1985;10:331-7.

15. Phillips $L H 2^{\text {nd }}$, Park TS. The frequency of intradural conjoined lumbosacral dorsal nerve roots found during seletive dorsal rhizotomy. Neurosurgery.1993;33:88-91.

16. Postacchini F, Urso S, Ferro L. Lumbosacral nerve root anomalies. J Bone Joint Surg. Am. 1982;64:721-9.

17. Prestar FJ. Anomalies and malformations of lumbar spinal nerve roots. Min Invasive Neurosurg. 1996;39:133-7.

18. Rask MR. Anomalous lumbosacral nerve roots in association with spondilolisthesis. Surg Neurol. 1977;8:139-40.

19. Savas R, Calli C, Yünten N, Alper H. Hypoplasic lumbar pedicle in association with conjoneid nerve root MRI demonstration. Comput Med Imag Graph. 1998;22:77-9.

20. Stambough J, Balderston RA, Booth RE, Rothman RH. Surgical management of sciatica involving anomalous lumbar nerve roots. J Spinal Disord. 1988;1:111-4.

21. Testut L. D'Anatomie humaine. $7^{\text {th }}$ ed. Paris: Gaston Doin; 1921. v. 3., p. 133-3.

22. White $3^{\text {rd }}$ JG, Strait TA, Binkley JR, Hunter SE. Surgical treatment of 63 cases of conjoined nerve roots. J Neurosurg. 1982;56:114-7.

Original recebido em julho de 2008

Aceito para publicação em março de 2009

Conflito de interesses não declarado

Endereço para correspondência

Max Franco de Carvalho

Rua José Seabra Batista, 22, Condomínio Tyrol, Edificio Innsbruck

49025-000 - Aracaju, SE, Brasil

E-mail:carvalhomax@hotmail.com 